ISSN (e)-2347-176x ISSN (p) 2455-0450

crossref DOI: https://dx.doi.org/10.18535/jmscr/v7i11.30

\title{
Clinical evaluation of MRI findings in chronic painful knee joint
}

\author{
Authors \\ Dr P Sai Prashanth Reddy ${ }^{1}$, Dr N Srinivas Reddy ${ }^{2 *}$ \\ ${ }^{1}$ Assistant Professor, Department of Orthopaedics, Prathima Institute of Medical Sciences, Nagunur, \\ Karimnagar, Telangana, India. \\ ${ }^{2}$ Associate Professor, Department of Orthopaedics, Prathima Institute of Medical Sciences, Nagunur, \\ Karimnagar, Telangana, India \\ *Corresponding Author \\ Dr N Srinivas Reddy
}

\begin{abstract}
Background: Any disruption of the intra-articular structures of the tri-compartmental knee joint or even extra-articular pathologies related to quadriceps mechanism and synovium can cause painful knee resulting in functional morbidity and disability. To ascertain and manage the root cause various modalities are available today. Magnetic Resonating Imaging (MRI) is an effective and non-invasive imaging modality in diagnosing and classifying the knee pathology.

Methods: The present study was a non-randomized prospective study conducted in the Department of Radiodiagnosis and Department of Orthopaedics, Prathima Institute of Medical Sciences, Nagunur, Karimnagar, Telangana, India. The study was carried out for a period of three years from January 2016 to January 2019. The study was done on patients presenting in the orthopedics outpatient department, Prathima Institute of Medical Sciences, with knee pain for the duration of more than 3 months and subsequent evaluation of the knee joint by using PHILIPSINGENIA 1.5 TESLA MRI Scanner. In each patient TIW, T2W, PD, STIR, GRE and PD-FAT SAT sequences are taken. A total of 100 patients with chronic knee pain i.e pain for the duration of more than 3 months, were studied.

Results: In our study, we found that meniscal tears in the young population (aged less than 40 years) and meniscal degeneration in patients above 40 years of age is the major finding. Grade 2 medial meniscal tears were most common followed by grade 3 medial meniscal tears, followed by lateral meniscal injury. ACL tears were the next most common finding after meniscal injuries or degeneration.

Conclusion: MRI is an accurate, non-invasive and cost-effective means to evaluate a painful knee. The high degree of precision in the interpretation of MR images and imaging in various planes and positioning the knee in $15-20^{\circ}$ of external rotation and 5-10 of flexion aided in delineating the site and the full extent of the lesions.

Keywords: Chronic Knee Pain, Menisci, Cruciate ligament, MRI.
\end{abstract}

\section{Introduction}

The value of magnetic resonance imaging (MRI) for imaging the knee was understood almost immediately after the introduction of this modality in the early 1980s. With the introduction of special closely coupled extremity coils, high field systems, open systems, extremity units, and other technical advances, the utility of MRI in the knee 
has expanded dramatically ${ }^{[1]}$. MR examination, a noninvasive modality, is now routinely used to assess a wide spectrum of internal knee derangements and articular disorders and has virtually replaced conventional arthrography in the evaluation of menisci and cruciate ligaments, decreasing both morbidity and costs associated with negative arthroscopic examinations. MR imaging has also proved valuable in the selection of surgical candidates and in preoperative planning $^{[2]}$. The decrease in the cost of MR knee studies has also contributed to their acceptance by the orthopedic community as a non-invasive replacement for arthrography and non-therapeutic arthroscopy ${ }^{[3]}$. A large number of disorders are best evaluated by MRI, including internal abnormalities such as those of cruciate ligaments and the menisci. Other disorders include abnormalities of patella and quadriceps mechanism, cartilage and synovium. Bone abnormalities such as osteonecrosis and tumors as well as bone contusions and fractures may also be detected. Other advantages offered by MRI examination are the absence of pain after-effects or morbidity ${ }^{[4]}$. The MRI examination poses almost no risk to the average patient. Fast spinecho imaging, used in conjunction with the fat suppression MR technique, has extended the sensitivity and specificity of MR for the detection of articular cartilage injuries. The three dimensional (3D) volume techniques have demonstrated the versatility of MR imaging in the evaluation of meniscal tears. It can be used to reformat images of meniscal tears in orthogonal and non-orthogonal planes. An additional advantage of MR imaging is multiplanar and thin section capabilities and the ability to evaluate the subchondral region and marrow. Chronic knee conditions like synovial arthropathies, synovial neoplasia, synovial osteochondromatosis, rare intra-articular abnormalities like lipoma arborescence, etc can be assessed satisfactorily with MRI as opposed to CT and other modalities.

\section{Material and Methods}

The present study was a non-randomized prospective study conducted in the Department of Radiodiagnosis and Department of Orthopedics, Prathima Institute of Medical Sciences, Nagunur, Karimnagar for a period of three years from January 2016 to January 2019. The study was done on patients presenting in orthopedics OPD, with knee pain for the duration of more than 3 months. These patients were referred to the Department of Radiodiagnosis for subsequent MRI evaluation of the knee joints. MRI evaluation was done by using PHILIPSINGENIA 1.5 TESLA Scanner. $\mathrm{N}=100$ patients with chronic knee pain i.e. pain for the duration of more than 3 months, were studied. In each patient T1W, T2W, PD, STIR, GRE and PD-FAT SAT sequences are taken. Typically, the patient is placed in a supine position with the knee placed in a closely coupled extremity coil. The knee was externally rotated 15 degrees to 20 degrees to facilitate visualization of ACL on sagittal images. The knee was flexed slightly (5 degrees to 10 degrees) to increase the accuracy of assessing the patella-femoral compartment and patellar alignment.

A form of $\mathrm{T} 2$ weighting (such as FS proton density [PD] FSE) was used in each of the three acquisition planes (axial, coronal and sagittal), for better visualization of Articular cartilage. A T2 gradient-echo (GRE) sagittal sequence was used to improve the accuracy of the detection of meniscal lesions by compensating for blurring inherent in most FSE acquisitions. Addition of short inversion time (TI) inversion recovery (STIR) sagittal acquisitions were also done to improve the identification of osseous contusions and muscle trauma. T2 GRE contrast imaging with intra-articular gadolinium was used to differentiate healing from repeated injury after primary meniscal repair or partial meniscectomy. All patients were screened for renal dysfunction by obtaining a history and laboratory kidney function tests before these contrast agents were used. The contrast was also used in suspicion of conditions like rheumatoid arthritis, osteomyelitis, 
and tumors. Delayed gadolinium-enhanced MRI cartilage (d GEMRIC) technique was used to identify early chondral lesions by assessing the amount of glycosaminoglycan in cartilage.

Mainly T1W axial, coronal and sagittal images are taken. An acquisition matrix (number of phase encodings) of 256 or higher, a field of view of 12 to $14 \mathrm{~cm}$, and 1 to 2 number of excitations (NEX) was routinely used. GRE images were acquired with an acquisition matrix of 192 or 256. For FS PD FSE images, TE values of 35 to $45 \mathrm{msec}$ were used to maintain a high SNR. Fast recovery FSE techniques were used with TR values of less than 3000 msec without compromising articular cartilage. STIR (fast inversion recovery) was done using a TR of $4000 \mathrm{msec}$, a TE of $18 \mathrm{msec}$, a TI of $140 \mathrm{msec}$ and an ETL (echo train length) of 4. Evaluation of neoplastic lesions, both benign and malignant, required a combination of $\mathrm{T} 1, \mathrm{~T} 2$ (conventional or FSE), STIR-(FS PD FSE were frequently used in place of STIR because of improved spatial resolution at higher field strengths). FS PD FSE or fast inversion-recovery sagittal or coronal images delineated the proximal-to-distal extent of the tumor on one complete image. Multiplanar imaging was done in all the cases and 4-millimeter sections were used for axial and coronal plane images, and 3-to 4-mm thick sections were used for sagittal images. The maximum slice thickness for the evaluation of the meniscus was $4 \mathrm{~mm}$. All the obtained data were recorded in the MS Excel spreadsheet and analyzed by statistical software.

\section{Results}

In this study, we studied $n=100$ patients over a period of 3 years. This included $n=76$ men and $\mathrm{n}=24$ women, $\mathrm{n}=47$ right knee joints and $\mathrm{n}=53$ left knee joints. The majority of the patients were in their fourth decade. The patients' age ranged from 16 years to 66 years with most of the patients in the age group of $31-40$ years.

Chart 1: showing the age-wise distribution of patients in the study

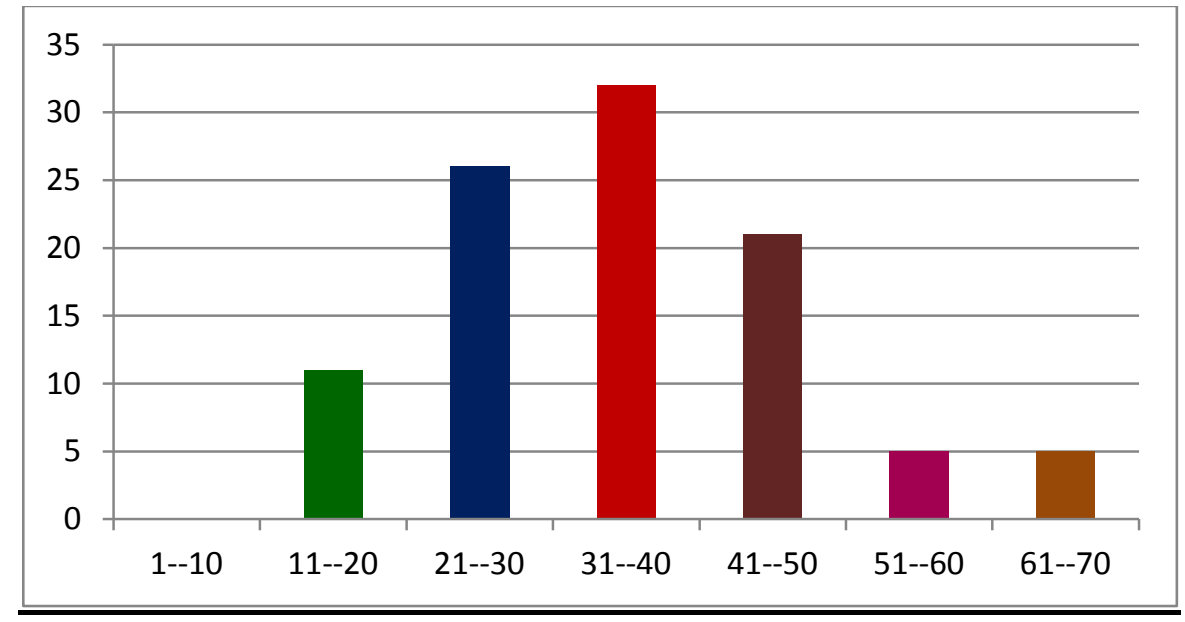

Out of these $n=100$ patients, 81 patients showed medial meniscal tears, $\mathrm{n}=53$ patients showed lateral meniscal tears, $n=55$ patients showed ACL tears and $\mathrm{n}=5$ patients showed PCL tears.
Following table 1 describes the various pathologies encountered in MRI evaluation of the patients. 
Table 2: Distribution of Knee Pathologies based on MRI

\begin{tabular}{|l|l|c|c|}
\hline $\begin{array}{l}\text { S1 } \\
\text { No }\end{array}$ & Abnormality & $\begin{array}{c}\text { Number of } \\
\text { Patients (N) }\end{array}$ & Percentage \\
\hline 1. & Anterior Cruciate Ligament Tears & 55 & 55 \\
\hline 2. & Posterior Cruciate Ligament Tears & 05 & 05 \\
\hline 3. & Medial Meniscal Tears & 81 & 81 \\
\hline 4. & Lateral Meniscal Tears & 53 & 53 \\
\hline 5. & Medial Collateral Ligament Tears & 07 & 07 \\
\hline 6. & Lateral Collateral Ligament Tears & 03 & 03 \\
\hline 7. & Chondromalacia Patellae & 03 & 03 \\
\hline 8. & Osteochondritis Dissecans & 02 & 02 \\
\hline 9. & Osteoarthritis & 30 & 30 \\
\hline 10. & Popliteal Cysts & 08 & 08 \\
\hline 11 & Osseous Fractures & 02 & 02 \\
\hline 12. & Rheumatoid Arthritis & 01 & 01 \\
\hline 13. & Infections & 01 & 01 \\
\hline 14. & Tumors & 02 & 02 \\
\hline 15. & Medial Patellar Retinaculum Tear & 02 & 02 \\
\hline 16. & Lateral Patellar Retinaculum Tear & 00 & 00 \\
\hline 17. & Joint Effusion & 26 & 87 \\
\hline 18. & Bone Marrow Edema & 26 \\
\hline
\end{tabular}

Medial Meniscal tears and Degeneration: Out of $n=100$ patients, $n=81$ patients showed medial meniscal tears and degeneration and 53 patients showed lateral meniscal tears and degeneration. Of $n=81$ patients with medial meniscal tears and degeneration, $\mathrm{n}=37(45.67 \%)$ showed grade II degeneration, $\mathrm{n}=33(40.74 \%)$ showed grade III degeneration, $\mathrm{n}=13(16 \%)$ showed grade I degeneration, $\mathrm{n}=1(1.23 \%)$ showed grade IV tear and $\mathrm{n}=2(2.40 \%)$ showed bucket handle tear.

Chart 2: Distribution of Medial Meniscal Grade III Tears

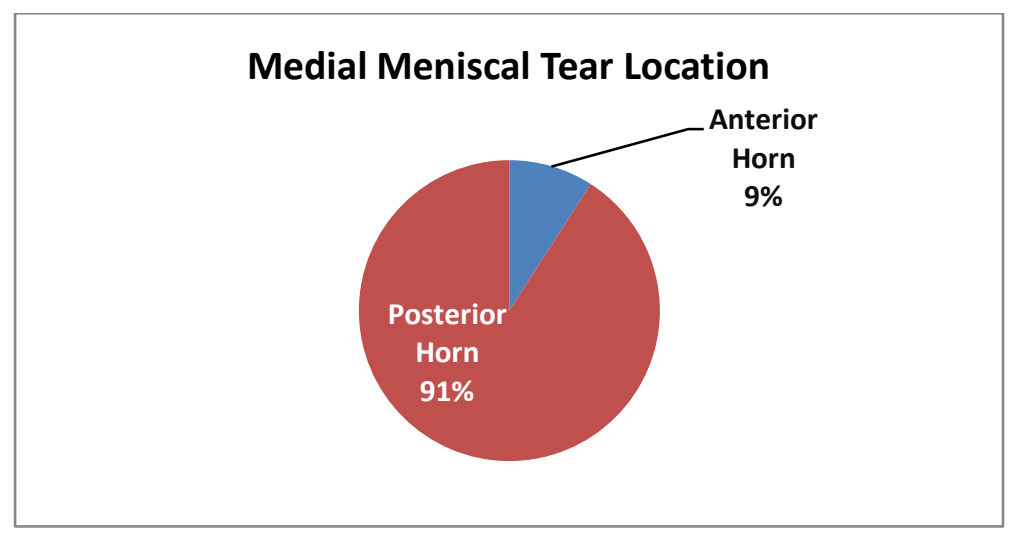

Lateral Meniscal Tears and Degeneration: Out of $n=53$ lateral meniscal tears and degeneration, $\mathrm{n}=23(43.39 \%)$ showed grade II degeneration, $\mathrm{n}=19(35.84 \%)$ showed grade III degeneration, $\mathrm{n}=14$ showed grade I degeneration, $\mathrm{n}=1 \quad(1.8 \%)$ showed grade IV degeneration and $\mathrm{n}=1(1.8 \%)$ showed bucket handle tear. 
Chart 3: Distribution of Medial Meniscal Grade III Tears

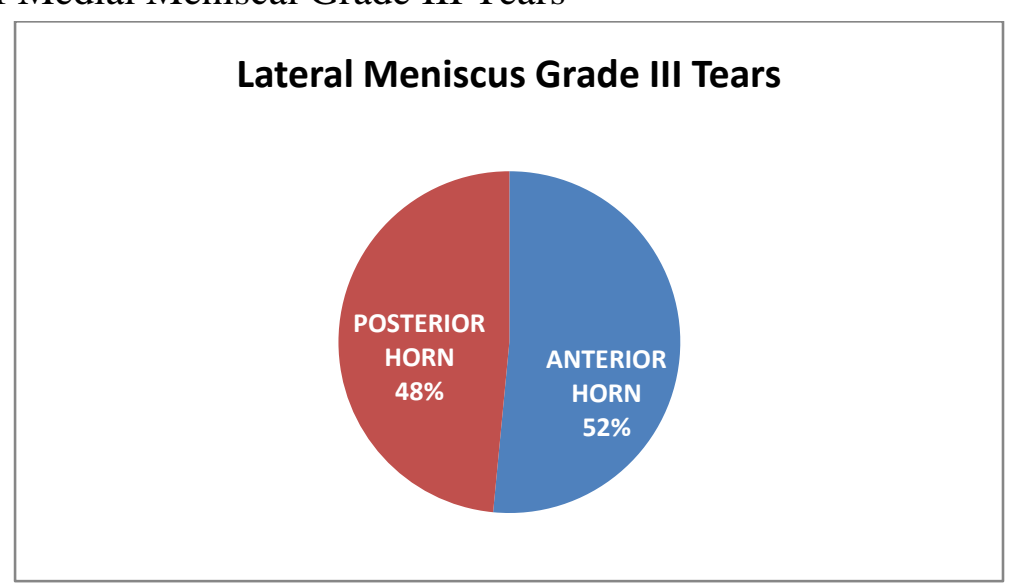

ACL tears and Degeneration: Out of $n=100$ patients, ACL tears were more common (55\%) than PCL tears which accounted for only $5 \%$. Out of 55 patients of ACL tears, $\mathrm{n}=28$ patients $(50.90 \%)$ had mid substance tears, $\mathrm{n}=21(38.18 \%)$ patients had tears at femoral attachment and $n=6$ patients $(10.90 \%)$ had torn at the tibial attachment, on MRI. In this study we found hyper-intensity in the ligament as the most common sign which was seen in $\mathrm{n}=52$ patients $(94.54 \%), \mathrm{n}=29(52.72 \%)$ patients showed discontinuity and $1(1 \%)$ patients showed non-visualization of ACL.

Table 3: Anterior Cruciate Ligament Tears

\begin{tabular}{|l|c|c|}
\hline Tears & Number $(\mathrm{N})$ & Percentage \\
\hline Complete & 23 & 41.82 \\
\hline Partial & 32 & 58.18 \\
\hline Total & 55 & 100 \\
\hline
\end{tabular}

Table 4: Location of ACL Tears

\begin{tabular}{|l|c|c|}
\hline ACL Tear & Number $(\mathrm{N})$ & Percentage \\
\hline Mid-substance & 28 & 50.90 \\
\hline Femoral Attachment & 21 & 38.18 \\
\hline Tibial Attachment & 06 & 10.90 \\
\hline
\end{tabular}

Chart 4: Primary signs of ACL Tear

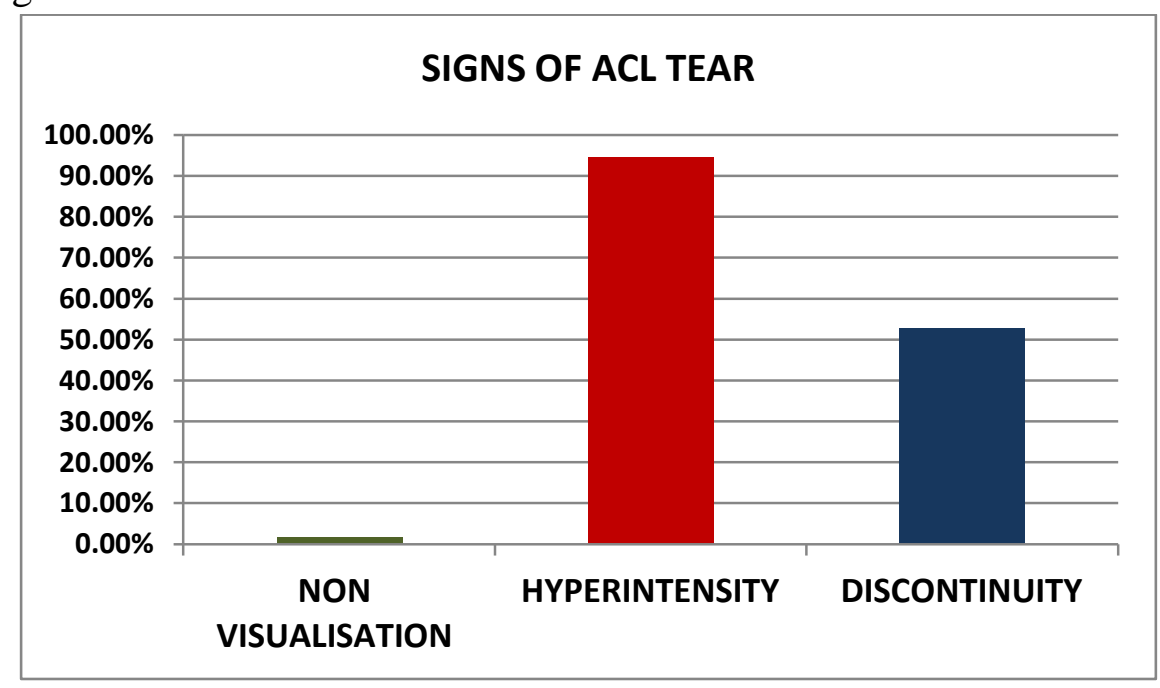


Table 5: Secondary Signs associated with ACL Tear

\begin{tabular}{|l|c|c|}
\hline Secondary Signs & Number (N) & Percentage \\
\hline Effusion & 51 & 92.72 \\
\hline PCL Buckling & 30 & 54.54 \\
\hline Anterior Tibial Subluxation & 00 & 00 \\
\hline Medial Collateral Ligament Tear & 03 & 5.4 \\
\hline Meniscal Tear & 32 & 58.18 \\
\hline Bone Bruise/Edema & 15 & 27.27 \\
\hline Segond's Fracture & 00 & 00 \\
\hline Uncovered Lateral Meniscus & 00 & 00 \\
\hline Deep Femoral Notch & 00 & 00 \\
\hline
\end{tabular}

Table 6: Distribution of Associated Injuries with PCL

\begin{tabular}{|l|c|c|}
\hline PCL Tear Associated Injuries & Number & Percentage \\
\hline Meniscal Tears & 04 & $80 \%$ \\
\hline ACL Tears & 05 & $100 \%$ \\
\hline Collateral Ligament Tears & 01 & $20 \%$ \\
\hline Haemarthrosis & - & \\
\hline Bone Contusion/Edema & 02 & $40 \%$ \\
\hline
\end{tabular}

Out of $\mathrm{n}=7$ patients with medial collateral collateral ligament tear, $\mathrm{n}=3(100 \%)$ had meniscal ligament tear, $n=5(71.42 \%)$ were found to have tears and $n=2(66.66 \%)$ had PCL tears. an ACL tear. Out of $n=3$ patients with lateral

Table 7: Distribution of Collateral Ligament Injuries

\begin{tabular}{|l|c|c|}
\hline & Number of Patients & Percentage \\
\hline COLLATERAL LIGAMENT INJURY \\
\hline Medial Collateral & 07 & $7 \%$ \\
\hline Lateral Collateral & 03 & $3 \%$ \\
\hline MCL TEAR ASSOCIATED INJURIES \\
\hline ACL Tears & 05 & $71.42 \%$ \\
\hline Bone Edema & 03 & $42.85 \%$ \\
\hline Meniscal Tear/Degeneration & 06 & $85.71 \%$ \\
\hline Fracture Of Tibial Plateau & - & - \\
\hline \multicolumn{2}{|c|}{ LCL TEAR ASSOCIATED INJURIES } \\
\hline MENISCAL TEARS & 03 & $100 \%$ \\
\hline PCL TEARS & 02 & $66.66 \%$ \\
\hline FIBULAR HEAD FRACTURE & - & \\
\hline
\end{tabular}

Table 8: Patellar Retinaculum Tears

\begin{tabular}{|l|c|c|}
\hline Patellar Retinaculum & Number & Percentage \\
\hline Medial & 03 & $75 \%$ \\
\hline Lateral & 01 & $25 \%$ \\
\hline Total & 4 & \\
\hline
\end{tabular}

Table 9: Popliteal Cysts

\begin{tabular}{|l|c|c|}
\hline POPLITEAL CYST & Number & Percentage \\
\hline UNRUPTURED & 7 & $87.50 \%$ \\
\hline RUPTURED & 1 & $12.50 \%$ \\
\hline TOTAL & 8 & \\
\hline
\end{tabular}


Chart 5: Other conditions found in MRI

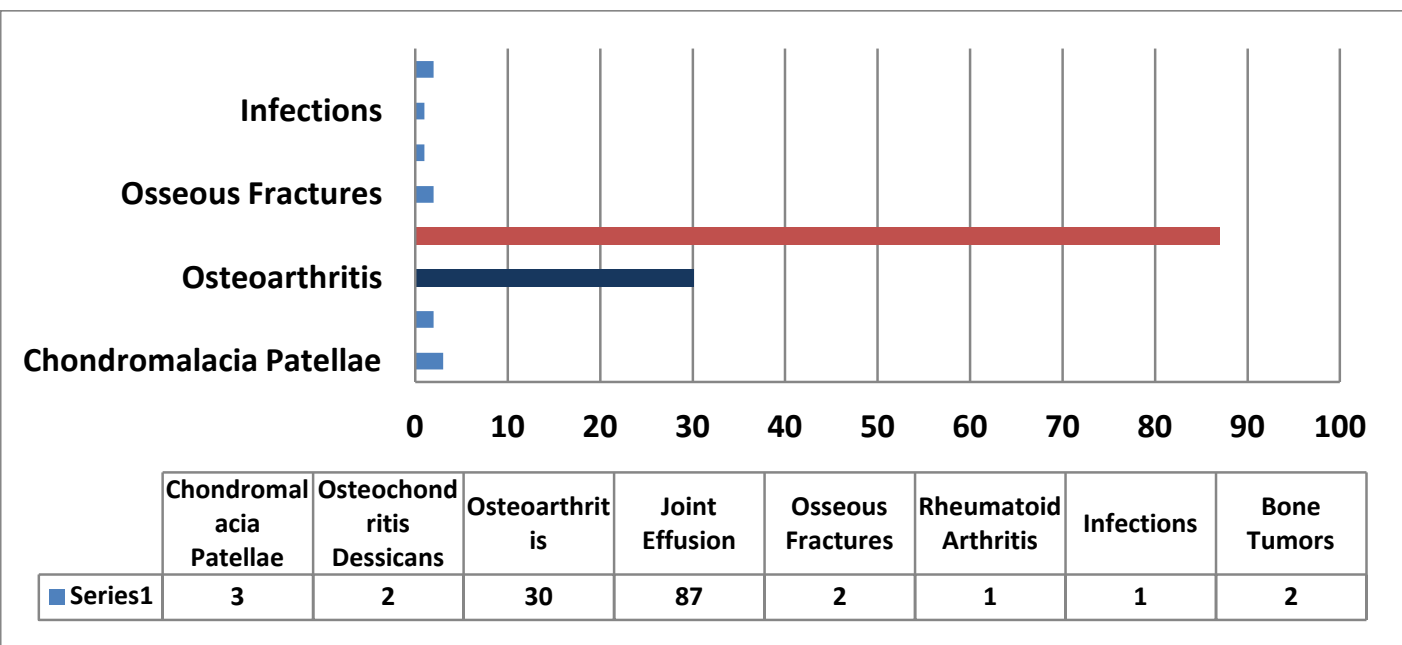

\section{Discussion}

In the present study out of $n=100$ patients, $n=81$ patients showed medial meniscal tears and degeneration and 53 patients showed lateral meniscal tears and degeneration. Medial meniscal tears and degeneration were more common $(81 \%)$ which corresponds with DS Shetty et al $;^{[5]}$ in their study on $n=115$ patients showed medial meniscal tears to be more common than lateral meniscus tears accounting for $36.5 \%$ case. Out of 81 medial meniscal tears and degeneration, $n=78$ involved the posterior horn and 11 involved the anterior horn. Stoller DW, et $\mathrm{al}^{[2]}$ in their study also showed posterior horn to be more frequently involved than the anterior horn. In our study, $n=3$ patients showed bucket handle tear, $n=2$ of which were in the medial meniscus and 1 in the lateral meniscus. This is corresponding with the study by Singson et $\mathrm{al}^{\left[{ }^{[6]}\right.}$ who reported that medial meniscus bucket handle tears are more common than the lateral meniscus. Out of three patients with bucket handle tear, two of them showed a double PCL sign. S Watts et al $;^{[7]}$ described that a double PCL sign is $98 \%$ specific but $32 \%$ sensitive. Out of $n=100$ patients, ACL tears were more common $(55 \%)$ than PCL tears which accounted for only $5 \%$. It contains a larger crosssectional area and possesses higher tensile strength, explaining its lower rate of injury. In this study we found hyper-intensity in the ligament as the most common sign which was seen in 52 patients (94.54\%), 29(52.72\%) patients showed discontinuity and $1(1 \%)$ patients showed no visualization of ACL. In our study, we found midsubstance as the most common location of tear and hyper-intensity as the most common sign of ligament tear. JP Singh et al ${ }^{[8]}$ in their study also reported mid-substance tear as the most common site and hyperintensity as the most common sign of a tear. Out of 55 cases of an ACL tear, 32 patients $(58.18 \%)$ were associated with a meniscal tear and 30 patients were associated with PCL buckling. McDaniel et al ${ }^{[9]}$ in their study reported that meniscal tear is associated with $85 \%$ to $91 \%$ of chronic ACL deficient knees. PCL tears accounted for a small number of cases $5(5 \%)$. Chernye et al ${ }^{[10]}$ stated that PCL is twice as strong as the ACL, with a large cross-sectional area and higher tensile strength thus accounting for a lower incidence of rupture of the PCL. The $n=7$ patients had medial collateral ligament injury and $n=3$ patients had lateral collateral ligament injury. In our study, the medial collateral ligament was more commonly injured than the lateral collateral ligament. David W. Stoller et al; ${ }^{[2]}$ stated that the lateral collateral ligament injury or disruption is less common than the injury to the medial collateral ligament. Out of $n=7$ patients with medial collateral ligament tear, 5(71.42\%) cases were found to have an ACL tear. Turek $\mathrm{SL}^{[11]}$ mentions that complete MCL rupture may be associated with tears of ACL. Out of 3 patients 
with lateral collateral ligament tear, 3 (100\%) had meniscal tears and 2(66.66\%) had PCL tears. According to Stoller DW et $\mathrm{al}^{[2]}$ injury or disruption of the LCL is significantly less common than the injury to the MCL. Also, he stated that LCL injuries may be seen in conjunction with either ACL or PCL injury. The findings on MRI were articular cartilage defects in medial patellar facet, with exposed subchondral bone and underlying fluid, appearing hypointense on T1WI and hyperintense on FS-PD weighted images and STIR images. Barry S. Yulish et al; ${ }^{[12]}$ in their study described that MR imaging is an accurate means of examining the posterior patellar cartilage and should be considered as an alternative to diagnostic arthroscopy when chondromalacia patellae are suspected. In our study, $\mathrm{n}=2(2 \%)$ patients showed osteochondritis dissecans. The findings on MRI were a hypointense signal on T1W, T2W and PDW images and hyperintense on STIR images, in the subchondral region of the anteromedial portion of the medial femoral condyle. $30 \%$ of patients had changes of osteoarthritis and showed a reduction in the medial compartment of the joint space, remodeling of the tibial and femoral condyle. Osteophytic changes were also seen in the patella, at femoral and tibial condyle along with tibial spiking. Krishanu B et al; ${ }^{[13]}$ described various MR findings in osteoarthritis of the knee. Our findings correlated with these; however chondral fragments and loose bodies were not seen. Also, other abnormalities associated with osteoarthritis were bone marrow edema, meniscal tears, effusion, baker cyst. This correlated with the study conducted by Peter R. Kornaat et al: ${ }^{[14]}$. In our study, one patient presented with rheumatoid arthritis. According to Gerhard Adam et $\mathrm{al}^{[15]}$ radiographs depict bony changes directly whereas disease of the articular cartilage, menisci, ligaments, and synovium are identified only indirectly. MR imaging has been proved to be useful in evaluating rheumatoid arthritis. Because of its high soft-tissue contrast, MR demonstrates cartilaginous, soft tissue and bone marrow abnormalities. In this study of 100 patients, $8(8 \%)$ of them showed popliteal cysts out of which, one was a ruptured cyst. These were associated with meniscal tears, joint effusion, and osteoarthritis. In a study conducted by John R Handy ${ }^{[16]}$ of 400 patients, $77(19 \%)$ were found to have popliteal cysts and a statistical correlation existed with effusion, meniscal tears, or "degenerative" arthropathy, or a combination of these 3 maladies ${ }^{[2]}$. In our study, two patients of tumors were studied, of which one was synovial sarcoma and the other was osteogenic sarcoma. D S Shetty et al in their study of 115 patients found a tumor in five patients and found that MR demonstrates internal hemorrhage, intraarticular, marrow, and soft tissue extension, with a great degree of accuracy. In this study, $2(2 \%)$ patients had a fracture of the intercondylar tibial tubercle. Stoller DW et al; ${ }^{[2]}$ in their study showed that fractures about the knee can involve the femoral condyle, tibial plateau, and patella. However, tibial plateau fractures are the most commonly seen. Out of 100 patients, 87 $(87 \%)$ cases had fluid accumulation in the central joint space and suprapatellar recess. Out of these cases, $48(55.17 \%)$ cases showing accumulation in the suprapatellar recess. This was consistent with the findings of Kaneko $\mathrm{K}$ et al ${ }^{[17]}$ stated that fluid preferentially accumulates in the suprapatellar recess and central portions of the joint in the traumatized knee. In our study, there was one case of chronic osteomyelitis. Carlos Pineda et $\mathrm{al}^{\left[{ }^{[18]}\right.}$ in their study showed that sensitivity and specificity of MRI are higher than those of plain radiography and CT; MRI is particularly good at depicting bone marrow abnormalities.

\section{Conclusion}

MRI is an accurate, non-invasive and costeffective means to evaluate a painful knee. The high degree of precision in the interpretation of MR images and imaging in various planes and positioning the knee in $15-20^{\circ}$ of external rotation and $5-10^{\circ}$ of flexion aided in delineating the site and the full extent of the lesions. An additional advantage of MR imaging is multiplanar and thin 
section capabilities and the ability to evaluate the subchondral region and marrow. As a result, MR imaging is recommended instead of plain radiograph and $\mathrm{CT}$ for evaluation of occult knee fractures, including tibial plateau fractures of the knee. The utility of MRI is enhanced by confirming that it is the appropriate imaging modality and that it is interpreted correctly. Judicious use of MRI also requires asking if there are another more cost-effective means to reach a diagnosis and if the results of MRI are likely to change the management of the problem.

\section{Conflict of interest: None \\ Source of support: Nil \\ Ethical Permission: Obtained}

\section{References}

1. Thomas H. Berquist. MRI of the Musculoskeletal System, $5^{\text {th }}$ ed. Lippincott Williams \& Wilkins 2006:422.

2. David W. Stoller, et al. Magnetic Resonance Imaging in Orthopedics and Sports Medicine, 3rd ed. Philadelphia: Lippincott Williams \& Wilkins 2007.

3. Nikken JJ, Oei EHG, Ginai AZ, et al. Acute peripheral joint injury: cost and effectiveness of low-field-strength MR imaging - results of a randomized controlled trial. Radiology 2005; 236:958-67.

4. C W HERON Review article: MRI of the knee. The British Journal of Radiology 1993; 66: 292-302.

5. DS Shetty, BN Lakhkar, GK Krishna. Magnetic Resonance Imaging in Pathologic Conditions of Knee. Ind J Radiol Imag 2002 12:3:375-381.

6. Singson RD, Feldman F, Staron R, Kiernan H. MR imaging of the displaced bucket handle tear of the medial meniscus. AJR 1991; 156: 121.

7. Watt S, Tonya H, Nigel R. The value of the absent Bowtie sign in MRI of Bucket handle tears. Clinical Radiology 2000; 55: 622-626.
8. JP Singh, L Garg et al. MR Imaging of knee with arthroscopic correlation in twisting injuries; Musculoskeletal 2004;14(1): 33-40.

9. Mc Daniel WJ. Untreated ruptures of anterior cruciate ligament.J Bone Joint Surg Am 1980; 62:696.

10. Chernye S. In Deer et al; Disorders of the knee.Principles of orthopedic practice, Vol 2.New York: Mcgraw- Hill 1989;128335.

11. Turek SL. Orthopedics: Principles and their applications, 4thed: Philadelphia JB Lippincott, 1984; 1269.

12. Barry S. Yulish, Josue Montanez, Donald B. Goodfellow, Patrick J. Bryan, George P. Mulopulos, Michael T. Modic. Chondromalacia Patellae: Assessment with MR Imaging. Radiology 1987; 164:763-766.

13. Krishanu B Gupta, Jeffery D, Barbara NW. Radiographic evaluation of osteoarthritis. Radiol Clin N Am 2004;42:11-41.

14. Peter R.Kornaat, Johan L.Bloem, Ruth Y.T.Ceulemans: Osteoarthritis of the knee: Association between Clinical Features and MR Imaging Findings. Radiology 2006; 239(3):811-817.

15. Adam G, Dammer M, Bohndrof K, Cristoph R, Fenke F Gunther RW. Rheumatoid arthritis of the knee: Value of gadopentetate dimeglumine-enhanced MR imaging. AJR Am J Roentgenol 1991 Jan;156(1):125-29.

16. John R Handy. Popliteal Cysts in Adults: A Review, Seminars in Arthritis and Rheumatism 2001; 31 (2): 108-118.

17. Kaneko K, De Money EH, Robinson AE. Distribution of joint effusion in patients with traumatic knee joint disorders: MRI assessment. Clin Imaging 1993; 17:176.

18. Carlos Pineda, Rolando Espinosa, Angelica Penca. Radiographic Imaging in Osteomyelitis: The Role of Plain Radiography, Computed Tomography, Ultrasonography, Magnetic Resonance Imaging, and Scintigraphy. Semin Plast Surg 2009; 23(2): 80-89. 